OPEN ACCESS

Edited by:

Michal Grivna,

United Arab Emirates University,

United Arab Emirates

Reviewed by:

DJavad Ghoddoosi-Nejad,

Birjand University of Medical

Sciences, Iran

Larry Kenith Olsen,

Logan University, United States

*Correspondence:

Qihui Lin

177791020@qq.com

Qing Chen

qch.2009@163.com

tThese authors have contributed equally to this work and share first

authorship

Specialty section:

This article was submitted to

Public Health Education and

Promotion,

a section of the journal

Frontiers in Public Health

Received: 08 May 2021

Accepted: 26 July 2021

Published: 20 August 2021

Citation:

Chen $H$, Zhang $M$, Su L, Cao H, Zhou X, Gu Z, LiU H, WU F, Li Q, Xian J, Chen $Q$ and Lin $Q$ (2021) Knowledge, Attitudes, and Practices

Toward COVID-19 Among Chinese Teachers, Shenzhen: An Online

Cross-sectional Study During the Global Outbreak of COVID-19.

Front. Public Health 9:706830.

doi: 10.3389/fpubh.2021.706830

\section{Knowledge, Attitudes, and Practices Toward COVID-19 Among Chinese Teachers, Shenzhen: An Online Cross-sectional Study During the Global Outbreak of COVID-19}

\author{
Hongbiao Chen ${ }^{1+}$, Minyi Zhang ${ }^{2 \dagger}$, Lixian Su ${ }^{3+}, \mathrm{He} \mathrm{Cao}^{1}$, Xiaofeng Zhou ${ }^{1}$, Zihao Gu ${ }^{1}$,

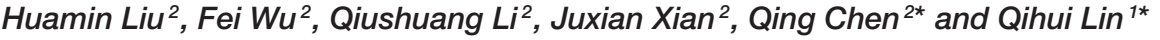 \\ ${ }^{1}$ Department of Epidemiology and Infectious Disease Control, Longhua Key Discipline of Public Health for the Prevention and \\ Control of Infectious Diseases, Longhua Centre for Disease Control and Prevention, Shenzhen, China, ${ }^{2}$ Department of \\ Epidemiology, School of Public Health, Southern Medical University, Guangzhou, China, ${ }^{3}$ Department of Child Healthcare, \\ Futian Maternity and Child Healthcare Hospital, Shenzhen, China
}

Background: Adequate understanding and precautionary behaviors are of vital importance to contain the spread of coronavirus disease 2019 (COVID-19). To date, the knowledge, attitudes, and practices (KAP) toward COVID-19 among different populations have been reported, whereas such information is unavailable in teachers. We aimed to investigate the KAP of teachers associated with COVID-19 during the global outbreak.

Methods: A large-scale population-based survey was conducted to gather information on COVID-19-related KAP among Chinese teachers using a self-administered questionnaire. We received 10,658 responses in April 2020, out of which 8,248 were enrolled in the final analysis. Participants responded to a self-administered questionnaire concerning demographic characteristics and KAP associated with COVID-19.

Results: This work included 4,252 (51.6\%) teachers in kindergartens, 2,644 (32.1\%) teachers in primary schools, and 1,352 (16.4\%) teachers in secondary schools. The knowledge level (mean: 4.46 out of seven points) was relatively lower than the levels of attitudes (mean: 3.27 out of four points) and practices (mean: 4.29 out of five points) toward COVID-19. Knowledge scores significantly varied by the collected demographic variables except education worksite $(p<0.05)$, whereas practice scores significantly differed in age groups $(p<0.05)$, education level $(p<0.001)$, education worksite $(p<0.001)$, and years of teaching $(p<0.001)$. The multivariate logistic analysis indicated that poor knowledge related to COVID-19 was common among men, younger, and less-educated teachers. In contrast, female teachers and those with higher education levels tend to have good practices against COVID-19. 
Conclusion: The present work suggested the knowledge gaps regarding COVID-19 were needed to be corrected immediately in teachers. Given the critical role of teachers in the education system, health authorities should take gender, age, and education level into account when developing suitable health interventions.

Keywords: COVID-19, knowledge, attitude, practice, teacher

\section{BACKGROUND}

The coronavirus disease 2019 (COVID-19), caused by severe acute respiratory syndrome-coronavirus-2 (SARS-CoV-2), has been spreading worldwide since December 2019 (1). In the light of the World Health Organization (WHO), more than 119.2 million confirmed cases of COVID-19 were reported as of March 9,2021 , resulting in great threats and significant challenges to human health (2). The number of COVID-19 cases is still rising, and thereby the fierce fight against the spread of COVID-19 is continuing across the globe (2). Since January 2020, many Chinese regions were locked down, and the Chinese residents were required to stay at home to avoid contact with others. Apart from the closure of numerous shops, markets, and public spaces, schools and universities were also suspended opening during the rapid rise period of the COVID-19 outbreak. Other countries had also imposed similar measures to make every possible effort to curb further transmission of COVID-19 inside and outside the nations (3). Public health emergencies affect the education, safety, and mental health of adolescents around the world. During the Ebola epidemic in West Africa, for instance, many children dropped out of school due to education interruption, as school closure would be helpful to curb the spread of Ebola (4). The current COVID-19 pandemic also forced most governments to temporarily close educational institutions, influencing more than $91 \%$ of the student population worldwide (4).

It is commonly recognized that good knowledge, attitudes, and practices (KAP) among the public are of vital importance to guarantee the final success of the battle against pandemics $(3,5)$. For instance, KAP surveys of the SARS in 2003 implied that knowledge and attitudes regarding infectious diseases are related to the level of panic emotion among persons, resulting in complicated attempts to epidemic prevention and control $(6,7)$. In the case of novel influenza A (H1N1) in 2009, the knowledge was not optimistic among the general Chinese population as most of them confused the transmission route of $\mathrm{H} 1 \mathrm{~N} 1$, providing evidence to the government for preventive measures during the H1N1 pandemic (8). Jalloh et al. indicated that Ebola-related KAP directly informed the development of a national social mobilization strategy concerning Ebola virus disease (9). Overall, KAP assessments are widely applied to identify knowledge gaps and individual behaviors among different sociodemographic

\footnotetext{
Abbreviations: KAP, knowledge, attitudes, and practices; COVID-19, Coronavirus disease 2019; SARS-CoV-2, severe acute respiratory syndrome-coronavirus-2; WHO, World Health Organization; SARS, severe acute respiratory syndrome; H1N1, the novel influenza A; CDC, Center for Disease Control and Prevention; $\mathrm{OR}$, odds ratio; $\mathrm{CI}$, confidence interval.
}

subgroups, providing effective interventions to improve public health $(10,11)$.

To date, a number of studies have been published focusing on COVID-19-related KAP within various populations, such as factory workers (12), general residents (5), income-poor households (13), medical-imaging professionals (14), university students (15), and healthcare workers (16). However, to the best of our knowledge, the COVID-19-related KAP has not been measured in the teacher population until now. As custodians of knowledge, the role of teachers is of paramount significance in the education system (17). A conclusion drawn from a review of seven published studies indicated that student mental health might be affected by their teachers (18). Another prior work reported that the attitudes of teachers and good practices had a crucial effect on their performance of the students (17). Hence, understanding the COVID-19-related KAP among the teachers is an urgent need at this critical moment.

In the present work, we conducted an online cross-sectional survey to determine the KAP related to COVID-19 among teachers engaged in kindergarten, primary, and secondary schools in China during the COVID-19 pandemic. Specifically, the purposes of our work included: (1) examining the levels of COVID-19-related KAP among the teacher population, (2) investigating the associations of KAP with demographic characteristics, and (3) identifying preferences of teachers regarding the sources of COVID-19 information in an attempt to facilitate health information acquisition.

\section{METHODS}

\section{Setting and Participants}

In April 2020, we conducted an online cross-sectional investigation using a self-administered questionnaire in Shenzhen City via the most extensive online survey platform in China called Wen Juan Xing (Changsha Ranxing Information Technology Co., Ltd., Hunan, China). We invited 24,000 teachers engaged in kindergartens, primary schools, and secondary schools covering the whole of Longhua, an urban district in Shenzhen city, to determine the status of COVID19-related KAP during the outbreak. Only the teachers who provided consent to be a part of the investigation were enrolled as participants. The initial data were collected from 10,658 individuals, with a response rate of $44.4 \%$. The exclusion criteria included the following: (1) duplicate questionnaires submitted by participants with the same IP address $(n=2,387)$ and (2) respondents who took $<100 \mathrm{~s}$ to complete the questionnaire $(n=23)$. A final total of 8,248 teachers without missing values were included in the present work (Supplementary Figure 1). 
The protocol for this work was approved by Longhua Center for Disease Control and Prevention (CDC), Shenzhen.

\section{Questionnaire}

A self-administered questionnaire was developed for the present survey. Based on the literature review regarding the given topic, CDC researchers designed and prepared the first version of the questionnaire. Subsequently, we conducted a pretest using 50 teachers other than those who were included in this work. After that, the first version of the questionnaire was reviewed again and modified by CDC researchers and experts in epidemiology and infectious diseases. The questionnaire consisted of four parts covering baseline information and KAP related to the COVID19 outbreak. Part I consisted of the general characteristics of the participants, including gender (male, female), age ( $\leq 30,31-$ $40,41-50$, and $>50$ years), education worksite (kindergarten, primary school, and secondary school), education levels (junior high school and below, high school, bachelor's degree, and master's degree or above), and years of teaching ( $\leq 2,3-5,6-$ 10 , and $>10$ years). Part II contained seven knowledge items to estimate the general understanding of clinical manifestation, transmission routes, and preventive measures associated with COVID-19 of the participants, including five multiple-choice items (K1-K5) and two single-choice items (K6-K7). For evaluation, a correct answer was given 1 point for each item, and otherwise, 0 point would be assigned according to the detailed scoring method, as described in Supplementary Table 1. The overall knowledge score ranged between 0 and 7 , and the participants were separated into two groups based on their knowledge scores. The "good" knowledge group consisted of participants who scored more than the total sample median (median $=5)$, whereas the "poor" knowledge group consisted of participants who scored below the total sample median. Part III focused on the attitudes toward COVID-19 estimated by four items (A1-A4) with the maximum possible score of 4. Likewise, the participants were divided into two groups. Participants who obtained a score more than the sample median $($ median $=3$ ) were regarded as indicating "good" attitudes, whereas those who scored below the median were regarded as indicating "poor" attitudes. Part IV included five items on practices (P1-P5) to estimate personal hygiene and behaviors during the pandemic. The maximum possible score was 5 . After calculating the practice scores, the participants were also divided into two groups. The "good" practice group included participants who obtained a score of more than the sample median (median $=4$ ), whereas a score below the median was assigned to a participant who was considered to denote "poor" practices. Similar to the work design of prior works (15), the knowledge section was developed based on the questions that have been used to assess COVID19-related knowledge levels $(5,12)$, whereas the attitude and practice sections were self-developed by researchers based on the scenarios most likely encountered by the teacher population for the present work. A summary of KAP items is shown in Supplementary Table 1.

Moreover, three additional questions on acquiring information related to COVID-19 were included as follows: (1) Is it necessary to be very familiar with the prevention and control guidelines about infectious diseases on campus? (Very necessary, necessary, and not necessary); (2) How do you prefer to obtain COVID-19-related information? [Internet or social software (WeChat, Weibo, and Zhihu), school physician, lectures, videos, and newspaper/poster]; (3) What information do you prefer to obtain concerning COVID-19? (Knowledge about the novel coronavirus, personal preventive measures, hygiene and disinfection, and no knowledge required) (Supplementary Figure 2). All questions were close-ended and treated as categorical variables.

\section{Statistical Analyses}

The categorical variables were presented as frequencies with percentages. The KAP scores of different persons, in the light of demographic variables, were described in the format mean \pm standard deviation. They were compared with independent samples $t$-test or one-way analysis of variance test for normal distribution data, which was determined by using histograms and Q-Q plots. Binary logistic regression analyses were carried out to investigate factors associated with COVID-19-related KAP by calculating odds ratios (OR) and 95\% confidence intervals (CI) after full adjustment for demographic variables, including gender, age, education level, education worksite, and years of teaching. Correlations between scores of KAP were also investigated, with Pearson correlation coefficients $(r)$ being obtained. All analyses were generated in R statistical software v.4.0.3 (R Foundation for Statistical Computing, Vienna, Austria). We considered statistical tests with $p$-values $<0.05$ statistically significant. All $p$-values were two-sided.

\section{RESULTS}

\section{General Characteristics of the Subjects}

Among all the 8,248 participants, most (89.2\%) were women, and over half the participants $(53.1 \%)$ were aged 30 years and younger; the group aged over 50 years accounted for only $3.7 \%$ of the participants. Among the participants, $40.7 \%$ attained a bachelor's degree, and $8.6 \%$ held a master's degree or above. Regarding educational institutions where the participants worked, 51.6\% worked in kindergartens, whereas 32.1 and $16.4 \%$ worked in primary and secondary schools, respectively. Also, $27.8 \%$ of them have been engaged in educational duties for $\leq 2$ years. Demographic characteristics are detailed in Table 1.

\section{COVID-19 Knowledge}

The accuracy rate of the seven items used to assess COVID19-related knowledge ranged from 8.5 to $85.7 \%$. For example, $79.4 \%$ of the participants correctly answered that the most common symptoms of COVID-19 included fever, fatigue, and dry cough. Most of the participants (85.6\%) correctly responded that wearing a mask and exercising during the pandemic can prevent COVID-19 infection. In comparison, few participants (8.5\%) correctly answered that vaccination was the safest, most effective, and economical measure against infectious diseases (Supplementary Table 1). The mean knowledge score was $4.46 \pm$ 1.25 points, indicating a total correct rate of $63.7 \%\left(4.46 / 7^{*} 100\right)$ on the present knowledge survey (Table 2). The knowledge 
TABLE 1 | General characteristics of participants, Shenzhen, China, April 2020.

\begin{tabular}{|c|c|c|c|}
\hline \multirow[t]{2}{*}{ General characteristics } & \multicolumn{2}{|c|}{ Total $(n=8,248)$} & \multirow[t]{2}{*}{$95 \% \mathrm{Cl}$} \\
\hline & $N$ & $\%$ & \\
\hline \multicolumn{4}{|l|}{ Gender } \\
\hline Male & 892 & 10.8 & $10.2-11.5$ \\
\hline Female & 7,356 & 89.2 & $88.5-89.8$ \\
\hline \multicolumn{4}{|l|}{ Age group (y) } \\
\hline$\leq 30$ & 4,379 & 53.1 & $52.0-54.2$ \\
\hline $31-40$ & 2,371 & 28.7 & $27.8-29.7$ \\
\hline $41-50$ & 1,192 & 14.5 & $13.7-15.2$ \\
\hline$>50$ & 306 & 3.7 & $3.3-4.1$ \\
\hline \multicolumn{4}{|l|}{ Education level } \\
\hline Junior high school or lower & 1,458 & 17.7 & $16.9-18.5$ \\
\hline High school & 2,723 & 33.0 & $32.0-34.0$ \\
\hline Bachelor's degree & 3,358 & 40.7 & $39.7-41.8$ \\
\hline Master's degree or above & 709 & 8.6 & $8.0-9.2$ \\
\hline \multicolumn{4}{|l|}{ Education worksite } \\
\hline Kindergarten & 4,252 & 51.6 & $50.5-52.6$ \\
\hline Primary school & 2,644 & 32.1 & $31.0-33.1$ \\
\hline Secondary school & 1,352 & 16.4 & $15.6-17.2$ \\
\hline \multicolumn{4}{|l|}{ Years of teaching } \\
\hline$\leq 2$ & 2,291 & 27.8 & $26.8-28.8$ \\
\hline $3-5$ & 2,286 & 27.7 & $26.8-28.7$ \\
\hline $6-10$ & 1,918 & 23.3 & $22.3-24.2$ \\
\hline$>10$ & 1,753 & 21.3 & $20.4-22.2$ \\
\hline
\end{tabular}

score significantly varied by gender $(p<0.001)$, age groups $(p<0.001)$, education levels $(p<0.001)$, and years of teaching $(p<0.001)$. Binary logistic analysis of COVID-19-related knowledge indicated that female teachers had a better knowledge of COVID-19 (OR 1.610, 95\% CI 1.380-1.879). Individuals aged 31-40 years (OR 1.540, 95\% CI 1.365-1.738) and 41-50 years (OR 1.823, 95\% CI 1.543-2.154) had more knowledge of COVID19 compared to those aged $\leq 30$ years. Moreover, participants with an educational level of high school were more likely to have good COVID-19-related knowledge (OR 1.220, 95\% CI 1.055-1.411) than those with a lower level of education. Teachers who held a bachelor's degree (OR 1.423, 95\% CI 1.196-1.694) or a master's degree (OR 1.350, 95\% CI 1.071-1.702) were also associated with good knowledge toward COVID-19 (Table 3).

\section{COVID-19 Attitudes}

The positive answer rate of the items on attitudes toward COVID-19 ranged between 45.7 and $98.2 \%$. Over $80 \%$ of the participants believed they could actively respond to the COVID19 pandemic despite feeling worried about it and could pay more attention to the prevention and control of infectious diseases in kindergartens or schools where they were. Nearly all the participants $(98.2 \%)$ indicated that they would not decrease vigilance against COVID-19, even though the situation is witnessing positive changes. However, more than half of the participants $(54.3 \%)$ felt pressure on working when the schools would reopen (Supplementary Table 1). The mean attitudes score was $3.27 \pm 0.71$ points out of 4 points, revealing a higher positive rate of $81.8 \%\left(3.27 / 4^{*} 100\right)$ on this series of attitude items (Table 2). At the same time, no statistical significance was observed between demographic variables and attitude scores. Results of the binary logistic analysis showed that participants teaching in secondary schools (OR 0.754, 95\% CI 0.588-0.966) were significantly associated with poor attitudes toward COVID19 vs. those in teaching in kindergartens. While having an education level of high school (OR 1.346, 95\% CI 1.0861.668) was a predictor of good attitudes compared with the education level of junior high school (Table 3), it should be noted that someone who did not attend high school but had obtained a kindergarten teacher certificate could be engaged as a kindergarten teacher in China.

\section{COVID-19 Practices}

Among our participants, the most frequent practice was staying at home $(95.3 \%)$ during the outbreak, followed by frequently washing hands with sanitizer (94.1\%) and following the standards for wearing a mask (85.5\%) during the period of COVID19. Most participants $(70.0 \%)$ have stored valuable articles such as sanitizer and drugs for further use against COVID19 (Supplementary Table 1). The mean practices score was $4.29 \pm 0.79$ points on the 5 -item scale, demonstrating that the best practice response rate was $85.8 \%\left(4.29 / 5^{*} 100\right)$ on this practice survey rather than investigation on COVID19-related knowledge and attitudes (Table 2). Practice scores significantly differed across age groups $(p=0.001)$, education levels $(p<0.001)$, education worksite $(p<0.001)$, and years of teaching ( $p<0.001)$. From the findings of the multivariate logistic analysis, female teachers (OR 1.465, 95\% CI 1.190-1.805) were more likely to have good practices responding to COVID19 than their male counterparts. Furthermore, individuals with higher education levels were significantly associated with good practices against COVID-19 ( $p$ for trend $<0.05$, junior high school or lower vs. master's degree or above, OR 2.397, 95\% CI 1.709-3.360) (Table 3).

There was a significant positive correlation between knowledge-practices $(r=0.136, p<0.001)$, knowledgeattitudes $(r=0.099, p<0.001)$, and attitudes-practices $(r=0.074, p<0.001)$ (Supplementary Table 2$)$.

\section{Access to Obtain COVID-19-Related Information}

The vast majority of the teachers (91.5\%) agreed that it is quite necessary to understand campus prevention and control guidelines for infectious diseases. Regarding sources of acquiring information related to COVID-19, most participants preferred to attend lectures organized by the government (61.1\%), followed by watching videos (55.9\%) and internet or social software (WeChat, Weibo, and Zhihu, 42.4\%). More than $70 \%$ of the participants were concerned about information in regard to the novel coronavirus, personal preventive measures, hygiene, and disinfection (Supplementary Figure 2). 
TABLE 2 | Knowledge, attitude, and practice scores of COVID-19 by demographic variables.

\begin{tabular}{|c|c|c|c|c|c|c|c|c|c|}
\hline Characteristics & \multicolumn{3}{|c|}{ Knowledge } & \multicolumn{3}{|c|}{ Attitude } & \multicolumn{3}{|c|}{ Practice } \\
\hline Gender & & -4.375 & $<0.001$ & & -1.103 & 0.270 & & -1.115 & 0.265 \\
\hline Male & $4.28 \pm 1.35$ & & & $3.25 \pm 0.71$ & & & $4.26 \pm 0.83$ & & \\
\hline Female & $4.49 \pm 1.23$ & & & $3.27 \pm 0.71$ & & & $4.29 \pm 0.79$ & & \\
\hline$\leq 30$ & $4.32 \pm 1.25$ & & & $3.28 \pm 0.71$ & & & $4.26 \pm 0.81$ & & \\
\hline $31-40$ & $4.63 \pm 1.21$ & & & $3.24 \pm 0.71$ & & & $4.34 \pm 0.77$ & & \\
\hline $41-50$ & $4.68 \pm 1.22$ & & & $3.29 \pm 0.69$ & & & $4.28 \pm 0.77$ & & \\
\hline$>50$ & $4.40 \pm 1.25$ & & & $3.30 \pm 0.68$ & & & $4.25 \pm 0.82$ & & \\
\hline Education level & & 8.745 & $<0.001$ & & 2.572 & 0.052 & & 32.236 & $<0.001$ \\
\hline Education worksite & & 0.953 & 0.386 & & 2.227 & 0.108 & & 20.948 & $<0.001$ \\
\hline Kindergarten & $4.45 \pm 1.23$ & & & $3.27 \pm 0.70$ & & & $4.23 \pm 0.80$ & & \\
\hline Primary school & $4.49 \pm 1.25$ & & & $3.28 \pm 0.71$ & & & $4.34 \pm 0.78$ & & \\
\hline Secondary school & $4.48 \pm 1.31$ & & & $3.23 \pm 0.73$ & & & $4.36 \pm 0.78$ & & \\
\hline Years of teaching & & 26.097 & $<0.001$ & & 0.303 & 0.823 & & 6.863 & $<0.001$ \\
\hline$\leq 2$ & $4.33 \pm 1.31$ & & & $3.26 \pm 0.71$ & & & $4.26 \pm 0.83$ & & \\
\hline $3-5$ & $4.41 \pm 1.24$ & & & $3.27 \pm 0.72$ & & & $4.25 \pm 0.80$ & & \\
\hline $6-10$ & $4.51 \pm 1.21$ & & & $3.26 \pm 0.70$ & & & $4.31 \pm 0.78$ & & \\
\hline$>10$ & $4.66 \pm 1.19$ & & & $3.28 \pm 0.70$ & & & $4.35 \pm 0.76$ & & \\
\hline
\end{tabular}

\section{DISCUSSION}

To the best of our knowledge, the present work is the first to investigate COVID-19-related KAP among teachers based on a large-scale sample size during the global outbreak of COVID19. Our findings indicated that the level of knowledge (mean score: 4.26 out of seven points) was relatively lower than the levels of attitudes (mean score: 3.27 out of four points) and practices (mean score: 4.29 out of five points) related to COVID-19.

At the time the survey was administered, most of the participants (79.4\%) correctly answered the most common clinical symptoms of COVID-19, including fever, fatigue, and dry cough. In prior studies targeting this item, the correct answer rate was $85.1 \%$ in both Syrian and South Korean residents $(3,10)$ and $96.4 \%$ in the general Chinese population regarding the main symptoms of COVID-19 (5). We considered that the different levels of this item might be due to the different item-format. In most previous studies, the items were answered on a yes/true/agree, no/false/disagree basis, with an additional option of not sure/do not know (3, 5, 10, 12, 14). In comparison, the format of multiple-choice items in the present work might indicate the fundamental knowledge level. In this case, $85.7 \%$ of the participants answered the three appropriate transmission routes of COVID-19; but however, $27.6 \%$ of them wrongly replied that the COVID-19 could be disseminated through mosquito bites (Supplementary Table 1).
This suggested that more education is needed to be provided by public health decision-makers and health-workers regarding the possible transmission routes of COVID-19.

In accordance with several surveys previously performed in China $(5,12)$, an overwhelming majority of our participants believed wearing a mask (99.7\%) and doing exercise (96.6\%) are adequate precautions to control and prevent the spread of COVID-19. However, wearing masks was recognized as an effective measure against COVID-19 by only $25.5 \%$ of the medical imaging professionals in India (14) and by $49.0 \%$ of income-poor households in the Philippines (13). These variations in knowledge levels might be attributed to the different precautions primarily implemented by countries. For the types of masks, most of the participants in the present work believed that N95 masks (90.8\%) and medical-surgical masks $(81.5 \%)$ are available to prevent COVID-19. However, we noted that some participants were confused about the appropriate types of the mask because they incorrectly answered dust masks (27.5\%), activated carbon masks (13.6\%), and cotton masks $(2.8 \%)$ in this item (Supplementary Table 1). During the early stages of COVID-19, the National Health Commission of China issued protocols for rapid precautions to effectively contain the spread of the epidemic (19). Simultaneously, the masks were introduced as a valuable personal protective equipment to slow down the transmission of the COVID-19 (20). This knowledge gap in wearing an appropriate mask was 
TABLE 3 | Factors associated with good knowledge, attitudes, and practices toward COVID-19 among the teacher population.

\begin{tabular}{|c|c|c|c|c|c|c|c|c|c|}
\hline \multirow[t]{2}{*}{ Characteristics } & \multicolumn{3}{|c|}{ Knowledge } & \multicolumn{3}{|c|}{ Attitude } & \multicolumn{3}{|c|}{ Practice } \\
\hline & Good (\%) & OR (95\% Cl) & $P$-value & Good (\%) & OR $(95 \% \mathrm{Cl})$ & $P$-value & Good (\%) & OR (95\% Cl) & $P$-value \\
\hline \multicolumn{10}{|l|}{ Gender } \\
\hline Male & $410(9.4)$ & 1.000 & & $778(10.8)$ & 1.000 & & $738(10.5)$ & 1.000 & \\
\hline Female & 3,949 (90.6) & $1.610(1.380-1.879)$ & $<0.001$ & 6,424 (89.2) & $0.952(0.758-1.194)$ & 0.668 & 6,287 (89.5) & 1.465 (1.190-1.805) & $<0.001$ \\
\hline \multicolumn{10}{|l|}{ Age group (y) } \\
\hline$\leq 30$ & $2,099(48.2)$ & 1.000 & & 3,838 (53.3) & 1.000 & & 3,696 (52.6) & 1.000 & \\
\hline $31-40$ & $1,383(31.7)$ & $1.540(1.365-1.738)$ & $<0.001$ & $2,043(28.4)$ & 0.907 (0.760-1.082) & 0.277 & 2,058 (29.3) & $1.175(0.993-1.392)$ & 0.061 \\
\hline $41-50$ & 724 (16.6) & $1.823(1.543-2.154)$ & $<0.001$ & $1,052(14.6)$ & $1.145(0.892-1.469)$ & 0.289 & $1,021(14.5)$ & $1.222(0.972-1.537)$ & 0.085 \\
\hline$>50$ & $153(3.5)$ & $1.294(0.988-1.695)$ & 0.061 & $269(3.7)$ & $1.142(0.760-1.716)$ & 0.523 & $250(3.6)$ & $0.974(0.683-1.390)$ & 0.886 \\
\hline \multicolumn{10}{|l|}{ Education level } \\
\hline Junior high school or lower & $744(17.1)$ & 1.000 & & $1,254(17.4)$ & 1.000 & & $1,181(16.8)$ & 1.000 & $\#$ \\
\hline High school & $1,394(32.0)$ & $1.220(1.055-1.411)$ & 0.007 & $2,413(33.5)$ & $1.346(1.086-1.668)$ & 0.007 & $2,271(32.3)$ & $1.250(1.034-1.511)$ & 0.021 \\
\hline Bachelor's degree & $1,856(42.6)$ & $1.423(1.196-1.694)$ & $<0.001$ & $2,919(40.5)$ & $1.274(0.986-1.646)$ & 0.064 & $2,941(41.9)$ & $1.880(1.482-2.384)$ & $<0.001$ \\
\hline Master's degree or above & $365(8.4)$ & $1.350(1.071-1.702)$ & 0.011 & $616(8.6)$ & $1.350(0.961-1.897)$ & 0.083 & $632(9.0)$ & $2.397(1.709-3.360)$ & $<0.001$ \\
\hline \multicolumn{10}{|l|}{ Education worksite } \\
\hline Kindergarten & $2,216(50.8)$ & 1.000 & & $3,736(51.9)$ & 1.000 & & $3,563(50.7)$ & 1.000 & \\
\hline Primary school & $1,414(32.4)$ & $0.943(0.830-1.072)$ & 0.373 & $2,307(32.0)$ & $0.878(0.724-1.065)$ & 0.187 & $2,286(32.5)$ & $0.906(0.755-1.086)$ & 0.285 \\
\hline Secondary school & $729(16.7)$ & $0.974(0.822-1.154)$ & 0.758 & $1,159(16.1)$ & $0.754(0.588-0.966)$ & 0.026 & $1,176(16.7)$ & $0.897(0.700-1.148)$ & 0.388 \\
\hline \multicolumn{10}{|l|}{ Years of teaching } \\
\hline$\leq 2$ & $1,143(26.2)$ & 1.000 & & $2,006(27.9)$ & 1.000 & & $1,920(27.3)$ & 1.000 & \\
\hline $3-5$ & $1,150(26.4)$ & $0.956(0.849-1.078)$ & 0.462 & $1,981(27.5)$ & $0.893(0.748-1.066)$ & 0.211 & $1,924(27.4)$ & $1.032(0.877-1.214)$ & 0.708 \\
\hline $6-10$ & $1,021(23.4)$ & $0.937(0.819-1.071)$ & 0.340 & $1,683(23.4)$ & $1.002(0.818-1.228)$ & 0.984 & $1,653(23.5)$ & $1.106(0.918-1.333)$ & 0.289 \\
\hline$>10$ & $1,045(24.0)$ & $1.025(0.861-1.219)$ & 0.783 & $1,532(21.3)$ & $0.972(0.751-1.256)$ & 0.826 & $1,528(21.8)$ & $1.113(0.871-1.421)$ & 0.392 \\
\hline
\end{tabular}

$\mathrm{OR}$, odds ratios; $\mathrm{Cl}$, confidence interval.

\#P for trend $<0.05$. 
an essential finding within the present work and needs to be corrected immediately.

We also asked the teachers a question about how to prevent and control the COVID-19 in classrooms. Most of the participants $(80.1 \%)$ fully answered that they agreed to keep the classrooms well-ventilated, disinfect items every day, and observe the body temperature of each student. Under the national guidelines, it is vital to perform body temperature monitoring to contain cluster cases of COVID-19 in educational institutions (19). However, when it came to an item pertaining to precautions to help prevent the spread of infectious diseases, most of our participants (91.5\%) failed to understand that vaccination was considered the safest, most effective, and most economical measure against COVID-19 $(21,22)$. Despite the fact that the development of COVID-19 vaccines had been progressing at an unprecedented rate (21), we presumed the insufficient knowledge about vaccinations against COVID-19 might be due to the lack of available vaccines as of April 2020. The National Health Commission of China officially included COVID-19 into the management of class B notifiable infectious diseases on January 20,2020 and followed class A notifiable infectious disease prevention and control steps $(23,24)$. Nevertheless, only $61.7 \%$ of our participants correctly responded that COVID-19 is a notifiable infectious disease in China (Supplementary Table 1). Hence, public health messaging of COVID-19 needs to be improved to deal with these knowledge gaps in the teacher population.

Female teachers showed a higher level of knowledge related to COVID-19 than men $(P<0.001)$. In line with previous work, women were more likely than men to listen to medical experts and extend their knowledge from the experiences of other countries and social media in response to COVID-19 (25). Compared with participants aged $<30$ years, those aged from 31 to 50 years had a significantly higher level of COVID19-related knowledge (both $p<0.001$ ). Also, the expected findings of better understanding of COVID-19 were observed in participants with a degree of high school $(P=0.007)$, bachelor's degree $(P<0.001)$, a master's degree, or above $(P=0.011)$ (Table 3). These highlighted that efforts should be directed toward increasing the knowledge of COVID-19 among men, younger, and less-educated teachers.

In agreement with attitude levels of COVID-19 documented among Chinese undergraduate students and the general Iranian population $(15,26)$, most of our work participants displayed positive attitudes toward COVID-19, illustrating that health education was effectively delivered throughout kindergartens and schools in Shenzhen. Interestingly, over half of the participants expressed their feeling of pressure to teach when schools reopen in the postpandemic era of COVID19 (Supplementary Table 1). Teachers from secondary schools showed a poor attitude about COVID-19 when compared with teachers working in kindergartens. The different educational situations could explain these findings among schools in China. In contrast to kindergartens, there are a larger number of students in the secondary schools, leading to much more complexity in management and teaching settings. Additional studies are needed to implement mental health exams among teachers in educational institutions. Moreover, the educational department should pay more attention to strengthening mental health care for the teachers and providing a more friendly educational environment.

The findings of COVID-19-related practices indicated that most participants (95.3\%) took precautions to prevent COVID19 infection by staying at home as much as possible during the outbreak, which was consistent with previous reports on COVID-19-related KAP in China $(5,12)$. This result could be primarily attributed to the strict prevention and control measures implemented by the Chinese governments in response to the severe situation, such as strict home quarantine, suspending public transportation, banning travel, and public gatherings, among other precautionary measures (3). As suggested by results of prior studies regarding gender patterns of risk-taking behaviors $(27,28)$, men have a greater likelihood of engaging in risk-taking behaviors. Meanwhile, a previous survey queried nearly 800 U.S. residents, and it showed that women had more tendency than men to embrace behaviors to prevent the spread of COVID-19, such as maintaining social distance, staying at home, and frequency of handwashing (29). The findings of our work were in accordance with these prior results that female teachers were significantly more likely to engage in good practices to help prevent the spread of COVID-19. As expected, our findings of a statistical trend between education levels and COVID-19related practices were generally consistent with previous reports $(5,12)$, indicating that higher education levels were found to be significantly correlated with a higher likelihood of engaging in good practices toward COVID-19 ( $p$ for trend $<0.05$ ). These results further suggested that health education intervention ought to be improved targeting specific demographic groups, especially for male teachers and those with lower education levels. Furthermore, our findings provided evidence of the significant positive correlations between knowledge-attitudes, knowledgepractices, and attitude-practices among the participants. These indicated higher COVID-19-related knowledge and attitudes scores associated with having good practices against COVID-19 in the present survey (Supplementary Table 2). These findings were in line with previous studies $(5,10)$, bringing to light that preventive behaviors could be heightened by improving their knowledge and attitudes toward COVID-19.

With respect to the source of COVID-19 information, most of our respondents indicated they would like to receive information through lectures, videos, and internet or mobile social software such as WeChat and Weibo. This result conformed to a prior KAP survey that targeted factory workers in China, indicating that new media, especially mobile social software, plays an essential role in disseminating public health messages associated with COVID-19 (12). Nonetheless, a cross-sectional survey among income-poor households in the Philippines showed that most participants preferred to acquire COVID-19 messages through traditional media sources, such as television and radio, and the limited internet connectivity in multiple areas in the Philippines supported this result $(13,30)$. Taken together, the use of public health communication strategies should be adapted to local conditions to provide the public with knowledge and guide them to take adequate measures when public emergencies arise. 
The strength of this survey, firstly, lies in the largescale population recruited during a critical period. Secondly, the format of multiple-choice items could reflect the actual knowledge level of the teachers related to COVID-19. Thirdly, the questionnaire was developed based on previous studies and through content validation. However, this work has several potential limitations that should be acknowledged. The targeted population only consisted of teachers from a single city that was not representative of all teachers in China. Given this critical moment, the information collected in this work might be subject to recall bias due to the use of self-reported questionnaires.

\section{CONCLUSION}

In summary, this large population-based work aimed at evaluating KAP toward COVID-19 among the teachers from kindergartens, primary schools, and secondary schools in Shenzhen, China. These results showed lower levels of COVID19-related KAP among men, younger, and less-educated teachers. Given the critical role of teachers in the education system, this work is expected to help health and education authorities formulate suitable interventions to address the knowledge gaps of teachers.

\section{DATA AVAILABILITY STATEMENT}

The raw data supporting the conclusions of this article will be made available by the authors, without undue reservation.

\section{REFERENCES}

1. Velavan TP, Meyer CG. Mild versus severe COVID-19: laboratory markers. Int J Infect Dis. (2020) 95:304-7. doi: 10.1016/j.ijid.2020.04.061

2. World Health Organization. Coronavirus Disease (COVID-2019) Situation Reports. Available online at: https://www.who.int/emergencies/diseases/ novel-coronavirus-2019/situation-reports (accessed March 15, 2021).

3. Al Ahdab S. A cross-sectional survey of knowledge, attitude and practice (KAP) towards COVID-19 pandemic among the Syrian residents. BMC Public Health. (2021) 21:1-7. doi: 10.1186/s12889-021-10353-3

4. United Nations Educational, Scientific and Cultural Organization. COVID-19 Educational Disruption and Response. Available online at: https://en.unesco. org/covid19/educationresponse (accessed April 8, 2020).

5. Zhong B-L, Luo W, Li H-M, Zhang Q-Q, Liu X-G, Li W-T, et al. Knowledge, attitudes, and practices towards COVID-19 among Chinese residents during the rapid rise period of the COVID-19 outbreak: a quick online cross-sectional survey. Int J Biol Sci. (2020) 16:1745. doi: 10.7150/ijbs.45221

6. Tao N. An analysis on reasons of SARS-induced psychological panic among students. J Anhui Instit Educ. (2003) 21:78-9. doi: 10.7416/ai.2021.2446

7. Person B, Sy F, Holton K, Govert B, Liang A. Fear and stigma: the epidemic within the SARS outbreak. Emerg Infect Dis. (2004) 10:358. doi: 10.3201/eid1002.030750

8. Lin Y, Huang L, Nie S, Liu Z, Yu H, Yan W, et al. Knowledge, attitudes and practices (KAP) related to the pandemic (H1N1) 2009 among Chinese general population: a telephone survey. BMC Infect Dis. (2011) 11:1-9. doi: 10.1186/1471-2334-11-128

9. Jalloh MF, Sengeh P, Monasch R, Jalloh MB, DeLuca N, Dyson M, et al. National survey of Ebola-related knowledge, attitudes and practices before the outbreak peak in Sierra Leone: August 2014. BMJ Global Health. (2017) 2:285. doi: 10.1136/bmjgh-2017-000285

\section{ETHICS STATEMENT}

The studies involving human participants were reviewed and approved by Longhua District Centre for Disease Control and Prevention. Written informed consent was not provided because we had obtain oral informed consent.

\section{AUTHOR CONTRIBUTIONS}

HCh, MZ, LS, and QLin contributed to design the framework of this work. HCa, XZ, and ZG contributed to the formal analysis. Data collection was performed by FW, QLi, and JX. The original draft of the manuscript was written by MZ and HCh. HL, QC, and QLin contributed to the manuscript review and editing. All authors have read and agreed to the published version of the manuscript.

\section{FUNDING}

This work was funded by the Science and Technology Innovation Funding Project of Shenzhen Longhua (grant number 2020206) and the Key Discipline of Control and Prevention of Infectious Diseases and Public Health of Shenzhen Longhua.

\section{SUPPLEMENTARY MATERIAL}

The Supplementary Material for this article can be found online at: https://www.frontiersin.org/articles/10.3389/fpubh. 2021.706830/full\#supplementary-material

10. Lee M, Kang B-A, You M. Knowledge, attitudes, and practices (KAP) toward COVID-19: a cross-sectional study in South Korea. BMC Public Health. (2021) 21:1-10. doi: 10.1186/s12889-021-10285-y

11. Papagiannis D, Malli F, Raptis DG, Papathanasiou IV, Fradelos EC, Daniil $\mathrm{Z}$, et al. Assessment of knowledge, attitudes, and practices towards new coronavirus (SARS-CoV-2) of health care professionals in Greece before the outbreak period. Int J Environ Res Public Health. (2020) 17:4925. doi: 10.3390/ijerph17144925

12. Li Z-H, Zhang X-R, Zhong W-F, Song W-Q, Wang Z-H, Chen Q, et al. Knowledge, attitudes, and practices related to Coronavirus disease 2019 during the outbreak among workers in China: a large cross-sectional study. PLoS Negl Trop Dis. (2020) 14:e0008584. doi: 10.1371/journal.pntd.0008584

13. Lau LL, Hung N, Go DJ, Ferma J, Choi M, Dodd W, et al. Knowledge, attitudes and practices of COVID-19 among income-poor households in the Philippines: a cross-sectional study. J Global Health. (2020) 10:11007. doi: 10.7189/jogh.10.011007

14. Kotian RP, Faujdar D, Kotian SP, D'souza B. Knowledge and understanding among medical imaging professionals in India during the rapid rise of the covid-19 pandemic. Health Technol. (2020) 10:1415-20. doi: 10.1007/s12553-020-00437-2

15. Peng Y, Pei C, Zheng Y, Wang J, Zhang K, Zheng Z, et al. A crosssectional survey of knowledge, attitude and practice associated with COVID19 among undergraduate students in China. BMC Public Health. (2020) 20:1292. doi: 10.1186/s12889-020-09392-Z

16. Zhang M, Zhou M, Tang F, Wang Y, Nie H, Zhang L, et al. Knowledge, attitude, and practice regarding COVID-19 among healthcare workers in Henan, China. J Hosp Infect. (2020) 105:183-7. doi: 10.1016/j.jhin.2020.04.012

17. Adu E, Olatundun S. Teachers' perception of teaching as correlates of students' academic performance in Oyo state, Nigeria. Essays Educ. (2007) 20:6. Available online at: https://openriver.winona.edu/eie/vol20/iss1/6 
18. Krane V, Karlsson B, Ness O, Kim HS. Teacher-student relationship, student mental health, and dropout from upper secondary school: a literature review. Scand Psychol. (2016) 3:e11. doi: 10.15714/scandpsychol.3.e11

19. National Health Commission of People's Republic of China. Notice on Printing and Distributing the Work Plan for Prevention and Control of Pneumonia Caused by Novel Coronavirus Infection in the Near Future. (2020). Available online at: http://www.nhc.gov.cn/tigs/s7848/202001/ 808bbf75e5ce415aa19f74c78ddc653f.shtml (accessed January 31, 2020).

20. Wei Q, Ren Z. Disinfection measures for pneumonia foci infected by novel coronavirus in 2019. Chin J Disinfect. (2020) 37:59-62.

21. Lurie N, Saville M, Hatchett R, Halton J. Developing Covid-19 vaccines at pandemic speed. N Engl J Med. (2020) 382:1969-73. doi: 10.1056/NEJMp2005630

22. Yang $Y$, Peng F, Wang $R$, Guan $K$, Jiang $T$, Xu G, et al. The deadly coronaviruses: The 2003 SARS pandemic and the 2020 novel coronavirus epidemic in China. J Autoimmun. (2020) 109:102434. doi: 10.1016/j.jaut.2020.102434

23. Adhikari SP, Meng S, Wu Y-J, Mao Y-P, Ye R-X, Wang Q-Z, et al. Epidemiology, causes, clinical manifestation and diagnosis, prevention and control of coronavirus disease (COVID-19) during the early outbreak period: a scoping review. Infect Dis Poverty. (2020) 9:1-12. doi: 10.1186/s40249-020-00646-x

24. National Health Commission of People's Republic of China. Pneumonia Infected With Novel Coronavirus Is Included in the Management of Legal Infectious Diseases. (2020). Available online at: http://www.nhc.gov.cn/jkj/ s7915/202001/e4e2d5e6f01147e0a8df3f6701d49f33.shtml (accessed January $31,2020)$.

25. Okten IO, Gollwitzer A, Oettingen G. Gender Differences in Preventing the Spread of Coronavirus. Behavioral Science \& Policy Association (2020).

26. Erfani A, Shahriarirad R, Ranjbar K, Mirahmadizadeh A, Moghadami M. Knowledge, attitude and practice toward the novel coronavirus (COVID-19) outbreak: a population-based survey in Iran. Bull World Health Organ. (2020) 30:10.2471. doi: 10.2471/BLT.20.25 6651
27. Pawlowski B, Atwal R, Dunbar R. Sex differences in everyday risktaking behavior in humans. Evol Psychol. (2008) 6:147470490800600104. doi: $10.1177 / 147470490800600104$

28. Cobey KD, Laan F, Stulp G, Buunk AP, Pollet TV. Sex differences in risk taking behavior among Dutch cyclists. Evol Psychol. (2013) 11:147470491301100206. doi: 10.1177/147470491301100206

29. New York University. Women More Likely to Embrace Behaviors Aimed at Preventing the Spread of COVID-19. ScienceDaily. (2020). Available online at: http://www.sciencedaily.com/releases/2020/10/201005092343.ht (accessed April 8, 2021).

30. Pastor CKL. Sentiment analysis on synchronous online delivery of instruction due to extreme community quarantine in the Philippines caused by COVID19 pandemic. Asian J Multidiscipl Stud. (2020) 3:1-6. Available online at: https://asianjournal.org/online/index.php/ajms/article/view/207

Conflict of Interest: The authors declare that the research was conducted in the absence of any commercial or financial relationships that could be construed as a potential conflict of interest.

Publisher's Note: All claims expressed in this article are solely those of the authors and do not necessarily represent those of their affiliated organizations, or those of the publisher, the editors and the reviewers. Any product that may be evaluated in this article, or claim that may be made by its manufacturer, is not guaranteed or endorsed by the publisher.

Copyright (c) 2021 Chen, Zhang, Su, Cao, Zhou, Gu, Liu, Wu, Li, Xian, Chen and Lin. This is an open-access article distributed under the terms of the Creative Commons Attribution License (CC BY). The use, distribution or reproduction in other forums is permitted, provided the original author(s) and the copyright owner(s) are credited and that the original publication in this journal is cited, in accordance with accepted academic practice. No use, distribution or reproduction is permitted which does not comply with these terms. 